\title{
Mott Transition, Ferromagnetism and Conductivity in the Generalized Hubbard Model
}

\author{
Yu. Skorenkyy, L. Didukh, O. Kramar \\ AND YU. DOVHOPYATY
}

Ternopil State Technical University, Ternopil, Ukraine

\begin{abstract}
The electron correlations in narrow energy bands are examined within the framework of the Hubbard model, generalized by taking into account the correlated hopping of electrons. Electronic conductivity and ferromagnetic ordering stabilization in the system with various forms of electronic density of states are studied. The influence of magnetic field, temperature and the form of density of states on concentration dependence of conductivity and magnetization is investigated. The correlated hopping is shown to cause the electron-hole asymmetry of transport and ferromagnetic properties of narrow band materials.
\end{abstract}

PACS numbers: 71.10.Fd, 71.27.+a

\section{Introduction}

Strongly correlated electron systems demonstrate unusual transport properties and peculiar magnetic behavior. Hubbard model [1] is the simplest model describing the essential physics of materials with strong electron correlations and is used intensively. Theoretical investigations of the conductivity in the Hubbard model last for many decades, by both analytical [2-4] and numerical $[5,6]$ methods. Extensive investigations of ferromagnetic properties of the model have been done (see $[7,8]$ and references therein). Recent advances enabled us to understand some of the peculiar properties, like metal-insulator transition under the action of external influences [9-11]. Results of great importance were obtained by the dynamical mean field theory (DMFT) in the limit of infinite spatial dimension [12]. Unfortunately, the analysis of DMFT is restricted to the symmetrical Hubbard model while for realistic models the non-local (dependent on wave vector) contributions to self-energy and transport characteristics are important, the case not covered by DMFT. At the same time, an adequate description of the electronic subsystem of many real transition metal compounds requires the generalization [13-16] 
of the Hubbard model by taking into account the correlated hopping of electrons (influence of electron concentration $n$ on the hopping processes). In such a model the hopping integrals, which describe translation movement of holes and doublons, differ one from the other. Interestingly, such a generalization can provide us with a new exactly solvable model $[17,18]$, which could serve as a test for the analytical and numerical methods.

In the present paper two-pole approximation [19] is used to study effects of electron correlations in the generalized Hubbard model. In Sect. 3 the single particle Green function and energy spectrum are obtained. The experimentally observable metal-insulator transitions are found in the model. In Sects. 4,5 ferromagnetic ordering stabilization and static conductivity of narrow-band material in the limit of strong Coulomb correlation are studied. The effect of temperature changes, doping, and magnetic field is investigated. The role of correlated hopping of electrons and the form of electronic density of states (DOS) is discussed.

\section{The effective Hamiltonian of a narrow energy band}

We write the Hamiltonian of correlated electron system as

$$
H=H_{0}+H_{1}+H_{1}^{\prime}+H_{\mathrm{ex}},
$$

where

$$
\begin{aligned}
& H_{0}=-\mu \sum_{i s}\left(X_{i}^{s}+X_{i}^{2}\right)+U \sum_{i} X_{i}^{2}+\mu_{\mathrm{B}} h \sum_{i s} \eta_{s} X_{i}^{s}, \\
& H_{1}=\sum_{i j s}^{\prime} t_{i j}(n) X_{i}^{s 0} X_{j}^{0 s}+\sum_{i j s}^{\prime} \tilde{t}_{i j}(n) X_{i}^{2 s} X_{j}^{s 2}, \\
& H_{1}^{\prime}=\sum_{i j s}^{\prime}\left(t_{i j}^{\prime}(n) \eta_{s} X_{i}^{s 0} X_{j}^{\bar{s} 2}+\text { h.c. }\right), \\
& H_{\mathrm{ex}}=-\frac{1}{2} \sum_{i j s}^{\prime} J(i j)\left[\left(X_{i}^{s}+X_{i}^{2}\right)\left(X_{j}^{s}+X_{j}^{2}\right)+X_{i}^{s \bar{s}} X_{j}^{\bar{s} s}\right] .
\end{aligned}
$$

Here Hubbard operator $X_{i}^{k l}$ describes transition of site $i$ from state $|l\rangle$ to state $|k\rangle, \mu$ is the chemical potential, $U$ is the energy of intra-site Coulomb repulsion of electrons, $J$ is the direct inter-site exchange interaction, $\mu_{\mathrm{B}}$ is the Bohr magneton, $h$ stands for the external magnetic field, $\eta_{s}=1$ for electron spin $s=\uparrow$ and -1 otherwise. Translation processes of holes and doublons are characterized by different hopping integrals, $t_{i j}(n)=\left(1-\tau_{1} n\right) t_{i j}$ and $\tilde{t}_{i j}(n)=\left(1-\tau_{1} n-2 \tau_{2}\right) t_{i j}$, respectively; $t_{i j}^{\prime}(n)=\left(1-\tau_{1} n-\tau_{2}\right) t_{i j}$ describes hoppings between single occupied and empty sites; correlated hopping parameters $\tau_{2}$ and $\tau_{1}$ describe the influence of sites involved into the hopping process and neighbor sites, respectively.

If we restrict ourselves to consideration of the strong correlation limit $(U \gg w(n))$ then at partial filling of the band the conductance is due to electron hopping within the Hubbard subbands and interband hopping can be neglected. At these conditions we apply the canonical transformation [20] to the Hamilto$\operatorname{nian}(1)$ 


$$
H_{\text {eff }}=\mathrm{e}^{S} H \mathrm{e}^{-S}
$$

with $S=\sum_{i j}\left[\frac{t_{i j}^{\prime}(n)}{U}\left(X_{i}^{\uparrow 0} X_{j}^{\downarrow 2}-X_{j}^{\downarrow 0} X_{i}^{\uparrow 2}\right)-\right.$ h.c. $]$. The operator $S$ is taken to exclude the processes with pair hopping of holes and doublons in the first order in the hopping parameter. Finally, we obtain the effective Hamiltonian

$$
H_{\mathrm{eff}}=H_{0}+H_{1}+H_{\mathrm{ex}}+\tilde{H}_{\mathrm{ex}},
$$

where

$$
\tilde{H}_{\mathrm{ex}}=-\frac{1}{2} \sum_{i j s}^{\prime} \tilde{J}(i j)\left(X_{i}^{s} X_{j}^{\bar{s}}-X_{i}^{s \bar{s}} X_{j}^{\bar{s} s}\right)
$$

with the indirect exchange interaction parameter $\tilde{J}(i j)=\left(t_{i j}^{\prime}(n)\right)^{2} / U$. This is the generalization of $t-J$ model [21] for systems with correlated hopping of electrons.

Using the variant of projection procedure in the case of $n<1$ we obtain

$$
E_{s}(\boldsymbol{k})=-\mu-z J n_{s}-z \tilde{J} n_{\bar{s}}+\alpha_{s} t_{\boldsymbol{k}}(n)+\beta_{s}
$$

for the single particle energy spectrum, where the correlated narrowing of the band and spin-dependent shift of subband center are

$$
\begin{aligned}
\alpha_{s} & =\frac{2-n+\eta_{s} m}{2}+\frac{n^{2}-m^{2}}{2\left(2-n+\eta_{s} m\right)}, \\
\beta_{s} & =-\frac{2}{\left(2-n+\eta_{s} m\right)} \sum_{\boldsymbol{k}} t_{\boldsymbol{k}}(n)\left\langle X_{i}^{\bar{s} 0} X_{j}^{0 \bar{s}}\right\rangle_{\boldsymbol{k}},
\end{aligned}
$$

respectively, and $z$ is the number of nearest neighbors to a site. The respective results for $n>1$ describe upper Hubbard subband

$$
\begin{aligned}
& \tilde{E}_{s}(\boldsymbol{k})=-\mu+U-z J n_{s}-z \tilde{J} n_{\bar{s}}+\tilde{\alpha}_{s} \tilde{t}_{\boldsymbol{k}}(n)+\tilde{\beta}_{s}, \\
& \tilde{\alpha}_{s}=\frac{n+\eta_{s} m}{2}+\frac{n^{2}-m^{2}}{2\left(n+\eta_{s} m\right)}, \quad \tilde{\beta}_{s}=-\frac{2}{\left(n+\eta_{s} m\right)} \sum_{\boldsymbol{k}} \tilde{t}_{\boldsymbol{k}}(n)\left\langle X_{i}^{s 2} X_{j}^{2 s}\right\rangle_{\boldsymbol{k}} .
\end{aligned}
$$

\section{Metal-insulator transition in the model}

To consider the metal-insulator transition (MIT) problem, we have to calculate the single-particle Green function for the case of intermediate correlation strength $U \simeq w(n)$ (here $w(n)$ denotes a half of band width). The functions $\left\langle\left\langle X_{p}^{\downarrow 2} \mid X_{s}^{2 \downarrow}\right\rangle\right\rangle$ and $\left\langle\left\langle X_{p}^{0 \uparrow} \mid X_{s}^{2 \downarrow}\right\rangle\right\rangle$ satisfy the equations of motion

$$
\begin{aligned}
& (E+\mu-U)\left\langle\left\langle X_{p}^{\downarrow 2} \mid X_{s}^{2 \downarrow}\right\rangle\right\rangle=\frac{\delta_{p s}}{2 \pi}\left\langle X_{p}^{\downarrow}+X_{p}^{2}\right\rangle+\left\langle\left\langle\left[X_{p}^{\downarrow 2}, H_{1}+H_{1}^{\prime}\right] \mid X_{s}^{2 \downarrow}\right\rangle\right\rangle, \\
& (E+\mu)\left\langle\left\langle X_{p}^{0 \uparrow} \mid X_{s}^{2 \downarrow}\right\rangle\right\rangle=\left\langle\left\langle\left[X_{p}^{0 \uparrow}, H_{1}+H_{1}^{\prime}\right] \mid X_{s}^{2 \downarrow}\right\rangle\right\rangle
\end{aligned}
$$

with $[A, B]=A B-B A$. To obtain the closed system of equations we apply the two-pole approximation [19]. Let us suppose in Eq. (6) that

$$
\left[X_{p}^{0 \uparrow}, H_{1}\right]=\sum_{j} \epsilon(p j) X_{j}^{0 \uparrow}, \quad\left[X_{p}^{\downarrow 2}, H_{1}\right]=\sum_{j} \tilde{\epsilon}(p j) X_{j}^{\downarrow 2},
$$

where $\epsilon(p j)$ and $\tilde{\epsilon}(p j)$ are non-operator expressions which we calculate using the 
method of paper [20]. At half-filling in a paramagnetic state we have

$$
\epsilon(p i)=\left(1-2 d+2 d^{2}\right) t_{p i}-2 d^{2} \tilde{t}_{p i}, \quad \tilde{\epsilon}(p i)=\left(1-2 d+2 d^{2}\right) \tilde{t}_{p i}-2 d^{2} t_{p i}
$$

with $d=\left\langle X_{p}^{2}\right\rangle$ being the concentration of doublons.

The functions $\left\langle\left\langle\left[X_{p}^{\downarrow 2}, H_{1}^{\prime}\right] \mid X_{s}^{2 \downarrow}\right\rangle\right\rangle$ and $\left\langle\left\langle\left[X_{p}^{0 \uparrow}, H_{1}^{\prime}\right] \mid X_{s}^{2 \downarrow}\right\rangle\right\rangle$ in Eq. (6) are taken into account in the mean-field approximation, in this way we neglect the processes describing the "inter-band" hoppings of electrons. Therefore, we close the system of equations and obtain Green function and the energy spectrum, exact in the band and atomic limits

$$
\begin{aligned}
& E_{1,2}(\boldsymbol{k})=-\mu+\frac{U}{2}+(1-2 d) \frac{t(\boldsymbol{k})+\tilde{t}(\boldsymbol{k})}{2} \\
& \mp \frac{1}{2} \sqrt{\left\{U-[t(\boldsymbol{k})-\tilde{t}(\boldsymbol{k})]\left(1-2 d+4 d^{2}\right)\right\}^{2}+\left[t^{\prime}(\boldsymbol{k})\right]^{2}},
\end{aligned}
$$

where $t(\boldsymbol{k}), t^{\prime}(\boldsymbol{k})$, and $\tilde{t}(\boldsymbol{k})$ are the Fourier transforms of hopping integrals $t_{i j}, t_{i j}^{\prime}$, and $\tilde{t}_{i j}$, respectively. Expression (10) describes the vanishing of the energy gap in the spectrum of paramagnetic insulator at critical value $\left(\frac{U}{w}\right)_{\mathrm{c}}$ when the half-band width $w$ increases (under pressure or doping). This expression also reproduces the exact result for a partial case of the model [18]. The energy spectrum (10) depends on temperature through the polar states concentration. For zero temperature and rectangular density of states some analytical expressions for $d$ can be derived. In the case of $\left|\varepsilon_{1,2}\right|>w$ where $\varepsilon_{1,2}$ are the roots of equation $E_{1,2}(\varepsilon)=0$ the concentration of polar states is found from the equation

$$
d=\frac{1}{4}-\frac{1}{4 w} \frac{\tau C}{D^{\frac{1}{2}}}\left(Q_{1}-Q_{2}\right)-\frac{U}{8 w} \frac{(1-\tau)^{2}}{D^{\frac{3}{2}}} \ln \left|\frac{D^{\frac{1}{2}} Q_{1}-D w-2 \tau U C}{D^{\frac{1}{2}} Q_{2}+D w-2 \tau U C}\right|,
$$

where $Q_{1,2}=\left[(U \mp 2 \tau C w)^{2}+(1-2 \tau)^{2} w^{2}\right]^{\frac{1}{2}}, D=4 \tau^{2} C^{2}+(1-\tau)^{2}, \tau=$ $\tau_{2} /\left(1-\tau_{1}\right), L_{1,2}=1 \mp D^{\frac{1}{2}} /[2(1-\tau)(1-2 d)]$. If $|\varepsilon|_{1,2}<w$ then we have

$$
d=\frac{1}{4}-\frac{U}{2 w}\left[\frac{\tau^{2} C^{2}}{(1-\tau)(1-2 d) D}+\frac{(1-\tau)^{2}}{4 D^{\frac{3}{2}}} \ln \frac{L_{1}}{L_{2}}\right] .
$$

The dependence $d\left(\frac{U}{w}\right)$ given by Eqs. (11), (12) is plotted in Fig. 1a. One can see that in the point $\left(\frac{U}{w}\right)_{\mathrm{c}}$ the slope of $d\left(\frac{U}{w}\right)$-dependence changes. Our result for $d\left(\frac{U}{w}\right)$ in region of MIT is in good agreement with result of papers [12, 22] obtained in the limit of infinite dimensions. Additionally, we have found that the correlated hopping reduces the doublon concentration and shifts the point of the cusp to the region of weak correlation. The dependence of the energy gap on temperature can lead to the transition from metallic to insulating state with an increase in temperature (in this connection let us note the transitions observed in the systems $\mathrm{NiS}_{2-x} \mathrm{Se}_{x}$ and $\left.\left(\mathrm{V}_{1-x} \mathrm{Cr}_{x}\right)_{2} \mathrm{O}_{3}\right)$. The application of the external pressure or other influences favoring electron hoppings (for example, a doping of anion subsystem) leads to the transition to a metallic state. 

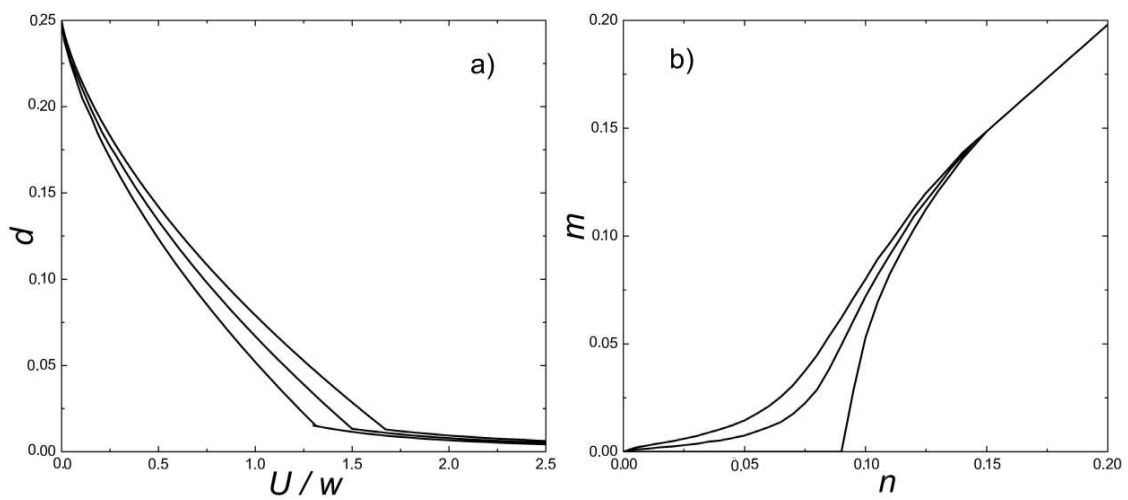

Fig. 1. (a) Polar states concentration as a function of the correlation strength parameter. Upper curve corresponds to $\tau_{2}=0$, middle curve $\tau_{2}=0.1$, lower curve $\tau_{2}=0.2$; (b) the concentration dependence of magnetization $m$ at $z J_{\text {eff }} / w=0$ ("tunable" DOS, $a=0.5$ ). Upper curve: $h / w=0.02$, middle curve $h / w=0.01$, lower curve $h / w=0$.

\section{Ferromagnetic ordering in the model: the influence of DOS form}

Let us calculate the ground state energy of the system described by effective Hamiltonian (3) in the case of $n<1$ :

$$
E_{0}=\frac{1}{2 N} \sum_{\boldsymbol{k} s} \int_{-\infty}^{\infty}\left[t_{\boldsymbol{k}}(n)+E\right] f(E) J_{\boldsymbol{k}}^{s}(E) \mathrm{d} E,
$$

where $f(E)$ is the Fermi distribution, $J_{\boldsymbol{k}}^{s}(E)$ is the spectral density of the Green function. We argue that a shape of non-interacting DOS (which corresponds to some lattice structure) substantially influences the critical electron concentration $n_{1}$, at which ferromagnetic ordering occurs as well as concentration $n_{2}$, at which magnetic moment becomes saturated. By the numerical calculations of the ground state energy on the base of expression (12) and subsequent minimization we have investigated the condition of ferromagnetism stabilization for various DOS. In particular, the numerical analysis has been done for DOS that corresponds to the simple cubic (sc) lattice [23], for DOS that corresponds to body-centered cubic (bcc) lattice [24] as well as for the "tunable" DOS $\rho(\epsilon)=c \sqrt{w^{2}-\epsilon^{2}} /(w+a \epsilon)$ with $c=\left(1+\sqrt{1-a^{2}}\right) /(\pi w)$. Changing the shape-controlling parameter $a$, one can modify DOS continuously and obtain both the semi-elliptical DOS $(a=0)$ and one with peak near the band-edge $(a \rightarrow 1)$, corresponding to a fcc lattice with next-nearest-neighbor hopping [25]. Our results $n_{1}=0.36$ and $n_{2}=0.62$ for sc lattice agree with the result of paper [26]. The spectral density approximation (SDA) $[27,28]$ gives the following results: $n_{1}=0.34, n_{2}=0.68$ for sc lattice and $n_{1}=0.52, n_{2}=0.68$ for bcc lattice. Our results for bcc lattice are $n_{1}=0.55$ and $n_{2}=0.64$ and also agree with paper [29]. In the case of "tunable" DOS with a peak near the band-edge we have $n_{1}=0.20$ and $n_{2}=0.31$ for $a=0.3$, $n_{1}=0.09$ and $n_{2}=0.15$ for $a=0.5$. With an increase in parameter $a$ the critical 
concentration of ferromagnetism onset decreases. Therefore, the existence of the peak near the band-edge in electronic DOS favors the ferromagnetic ordering in accordance with Ref. [25]. In the case of strong electron correlation at $n=1$ (when the shifts of subband center vanish) the ferromagnetic ordering is stabilized only by the interatomic exchange (independently of DOS used). At semi-elliptical DOS the saturated ferromagnetic state above $n=0.59$ is realized. If $z J_{\text {eff }} / w>0$ then the stability of ferromagnetic state rises because the energy difference of the paramagnetic and ferromagnetic ground states increases, otherwise ordering is realized due to spin-dependent shifts of subband center. The correlated hopping leads to the narrowing of the band and suppresses the "translational" mechanism of ferromagnetism. In the magnetic field the changes of magnetization become smooth, the value of $m$ depends on the chosen DOS form and correlated hopping substantially (Fig. 1b).

\section{Electronic conductivity in the strong interaction limit}

With the use of the method of papers [30,31], we calculate the $x x$-component of static electronic conductivity

$$
\sigma=\frac{e^{2} \tau z}{2 N a}\left(\sum_{i j s} t_{i j}(n)\left\langle X_{i}^{s 0} X_{j}^{0 s}\right\rangle+\sum_{i j s} \tilde{t}_{i j}(n)\left\langle X_{i}^{2 s} X_{j}^{s 2}\right\rangle\right),
$$

where the first sum is the conductivity of lower $(0-s)$-subband, the second sum is the conductivity of upper $(\uparrow \downarrow-\bar{s})$-subband. Here the magnetization of the system is an important parameter which is calculated by the method of Sect. 4 separately for $n<1$ and $n>1$. As one can see from Fig. 2a, due to the correlated hopping of electrons the conductivity provided by carriers in upper subband is substantially lower than the conductivity, provided by carriers from lower subband. This effect is a manifestation of the electron-hole asymmetry, inherent to real transition metal compounds. Other important feature is the change of current carrier type from metallic to semiconducting type in the vicinity of $n=2 / 3,4 / 3$ and from semiconducting type to metallic one at $n=1$ at an increase in band filling. The increase in the correlated hopping will shift maxima of the conductivity closer to the half-filling. External magnetic field changes the concentration dependence of $\sigma$ qualitatively (see Fig. 2b). The higher is the electron concentration, the less pronounced is the effect of applied magnetic field. At a small concentration of electrons the band is fully polarized, in such a ferromagnetic system the conductivity is considerably lower than in the paramagnetic state. If electron concentration rises, the decrease in magnetization leads to the increase in conductivity, $\sigma$ approaches its value in the paramagnetic state. The position of conductivity maximum changes from $n=2 / 3$ in the saturated ferromagnetic state (Fig. 2b, middle curve) to $n=0.5$ in the ferromagnetic state (Fig. 2b, lower curve). The form of electronic density of states has a substantial effect on the concentration dependence of static conductivity (Fig. 3a). At simple rectangular 


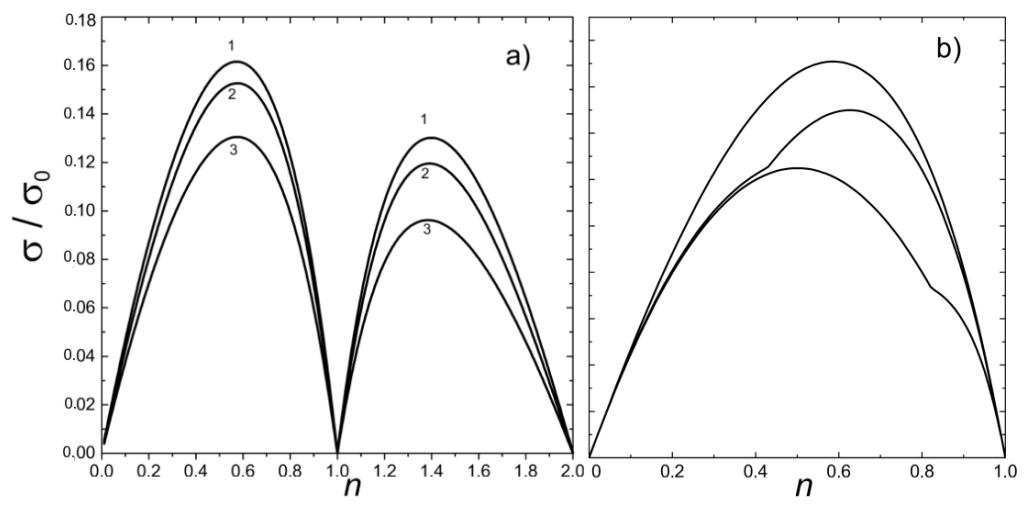

Fig. 2. (a) The concentration dependences of static electronic conductivity for values of the correlated hopping $\tau_{1}=\tau_{2}=0.1$. Curve 1 corresponds to the temperature $\Theta / w=0.01$, curve 2 to $\Theta / w=0.1$, curve 3 to $\Theta / w=0.2$; (b) the concentration dependences of the electronic conductivity in the magnetic field at temperature $\Theta / w=0.02, z J_{\text {eff }} / w=0.02, \tau_{1}=\tau_{2}=0$. The upper curve corresponds to $h / w=0$, the middle one to $h / w=0.005$, the lower curve to $h / w=0.02$.
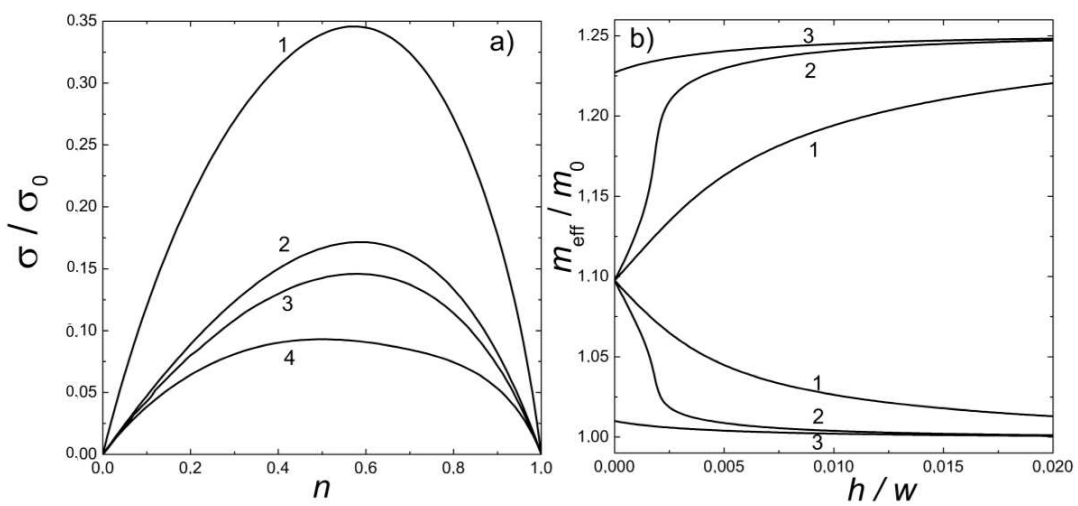

Fig. 3. (a) The concentration dependences of conductivity at various DOS forms. Curve 1 corresponds to sc lattice, curve 2 corresponds to the rectangular DOS, curve 3 to semi-elliptical DOS, and 4 to bcc lattice. (b) The effective mass of current carriers with spin up (upper curves) and down (lower curves) as a function of the external magnetic field. Curves 2 correspond to $z J_{\text {eff }} / w=0, \Theta / w=0.02$, curves 1 to $z J_{\text {eff }} / w=0$, $\Theta / w=0.05$, curves 3 to $z J_{\text {eff }} / w=0.05, \Theta / w=0.02$.

DOS the maximum of the conductivity in the lower Hubbard band corresponds to $n=2 / 3$, while for semi-elliptical DOS, one with asymmetry [32] with respect to the band center, DOS for simple cubic or body-centered cubic lattices the maximum of conductivity moves. This can be explained by the behavior of kinetic energy at change of electron concentration for various forms of DOS. Concentra- 
tion dependence of kinetic energy and effect of correlation (through the correlation narrowing of the band and effective mass enhancement) on the position of ground energy minimum (and thus conductivity maximum) allows us to interpret the differences of conductivity concentration dependences for various forms of DOS. We have obtained that effective mass of heavy carriers can increase substantially with the increase in electron concentration. Besides, in the paramagnetic states the carriers in upper subband with masses $\tilde{m}_{\text {eff }}=\hbar /\left(2 a^{2}|\tilde{t}(n)|\right)$ are much heavier than the carriers in lower subbands with masses $m_{\text {eff }}=\hbar /\left(2 a^{2}|t(n)|\right)$, the effective mass increases stepwise at the point $n=1$. The rise in magnetization leads to the rise of difference in effective masses of spin-up and spin-down current carriers (Fig. 3b). The results shown in Fig. 3b qualitatively agree with the corresponding plot of paper [33], where the Gutzwiller approximation has been used to calculate effective masses of current carriers. The correlated hopping, favoring localization, shifts effective masses up. Different possible scenarios of $m_{\mathrm{eff}}(h)$ dependence are shown in Fig. 3b. At high temperature $m_{\text {eff }}$ changes monotonically while at low temperature the system goes unstable towards the transition to polarized state. Direct exchange interaction can stabilize ferromagnetically polarized state in the less than half-filled band even in a weak magnetic field.

\section{Discussion and conclusion}

In this paper we have used a model with correlated hopping of electrons to study the metal-insulator transition, ferromagnetic ordering, and static conductivity of Mott-Hubbard material. The single-electron Green function and quasi-particle energy spectrum of the model have been calculated using a variant of the generalized Hartree-Fock approximation. These allow us to describe metalinsulator transitions as well as stabilization of magnetic orderings in the generalized Hubbard model (with electron-hole asymmetry). In the ferromagnetic state the translational shifts of the spin subband centers have been calculated self-consistently and appear to depend substantially on the DOS form. Numerical calculation of the ground state energy at different forms of DOS corresponding to some types of crystal lattice has been performed, magnetization and critical concentration for ferromagnetism stabilization has been found.

The concentration dependence of $\sigma$ has peculiarities inherent to transition metal compounds. We found the changes of current carrier type around $n=2 / 3,1,4 / 3$. In the limiting case of the absence of correlated hopping our results for the static conductivity agree with the concentration dependences obtained in the composite operator method [3], exact diagonalization [5], and Monte Carlo simulation [6] results. In the magnetic field static conductivity reflects the changes of single electron energy spectrum through correlation narrowing of the band and shift of subband center. The changes of concentration dependences of magnetization at changes of temperature lead to $\sigma(n)$ dependence with maxima at quarter and three-quarter fillings in distinction with paramagnetic ones. Effective masses 
of quasi-particles appear to be spin-dependent and substantially vary with magnetic field. These results are in agreement with the analysis of papers [33, 34] and experimental data [35] for heavy-fermion compounds.

We argue that the correlated hopping leads to the energy bands narrowing and insulating state stabilization, the decrease in shifts of spin-subbands centers and suppresses the translational mechanism of ferromagnetic ordering and leads to the increase in effective masses. The form of electronic density of states has a substantial effect on concentration dependences of both static conductivity and system magnetization. Taking into account the correlated hopping, which is inherent to real narrow band materials, allows us to describe electron-hole asymmetry which is observed in real materials.

\section{Acknowledgments}

Authors are grateful to Prof. J. Spałek and Prof. I.V. Stasyuk for the enlightening discussions. Partial support from Ukrainian Fund for Fundamental Research and Marie Curie TOK program is gratefully acknowledged.

\section{References}

[1] J. Hubbard, Proc. R. Soc. A 276, 238 (1963).

[2] W. Brenig, Z. Phys. 89, 187 (1992).

[3] W. Mancini, D. Villiani, Phys. Lett. A 261, 357 (1999).

[4] J.E. Hirsch, Phys. Rev. B 59, 6256 (1999); ibid. 62, 14131 (2000).

[5] E. Dagotto, Rev. Mod. Phys. 66, 763 (1994).

[6] D.J. Scalapino, S.R. White, S.C. Zhang, Phys. Rev. Lett. 68, 2830 (1992).

[7] P. Fazekas, Lecture Notes on Electron Correlation and Magnetism, World Scientific, Singapore 1999.

[8] Band-Ferromagnetism. Ground State and Finite-Temperature Phenomena, Eds. K. Babershke, M. Donath, W. Nolting, Springer, Berlin 2001.

[9] N.F. Mott, Metal-Insulator Transitions, Taylor and Francis, London 1990.

[10] M. Imada, A. Fujimori, Y. Tokura, Rev. Mod. Phys. 70, 1039 (1998).

[11] J.M. Honig, J. Spałek, Chem. Mater. 10, 2910 (1998).

[12] A. Georges, G. Kotliar, W. Krauth, M. Rozenberg, Rev. Mod. Phys. 68, 13 (1996).

[13] L. Didukh, Fiz. Tverd. Tela 19, 1217 (1977) (in Russian).

[14] S. Kivelson, W.-P. Su, J.R. Schrieffer, A.J. Heeger, Phys. Rev. Lett. 58, 1899 (1987).

[15] R. Micnas, J. Ranninger, S. Robaszkiewicz, Phys. Rev. B 39, 11653 (1989).

[16] H.Q. Lin, J.E. Hirsch, Phys. Rev. B 52, 16155 (1995).

[17] A. Montorsi, M. Rasetti, Phys. Rev. Lett. 66, 1383 (1991).

[18] L. Arrachea, A.A. Aligia, Phys. Rev. Lett. 73, 2240 (1994).

[19] L. Didukh, Acta Phys. Pol. B 31, 3097 (2000). 
[20] L. Didukh, J. Phys. Stud. 1, 241 (1997) (in Ukrainian).

[21] K.A. Chao, J. Spalek, A.M. Oles, J. Phys C 10, L271 (1977).

[22] G. Kotliar, M. Rozenberg, in: The Hubbard Model, Ed. D. Baeriswyl, Plenum Press, New York 1995, p. 155.

[23] T. Tonegava, Prog. Theor. Phys. 56, 1293 (1974).

[24] J. Jelitto, J. Phys. Chem. Solids 30, 609 (1969).

[25] D. Vollhardt, N. Blümer, K. Held, M. Kollar, J. Schlipf, M. Ulmke, Z. Phys. B 103, 283 (1997)

[26] L. Roth, Phys. Rev. 184, 451 (1969).

[27] G. Gaipel, W. Nolting, Phys. Rev. B 38, 2608 (1988).

[28] T. Hermann, W. Nolting, Solid State Commun. 103, 351 (1997).

[29] A.V. Zarubin, V.Yu. Irkhin, Fiz. Tverd. Tela 41, 1057 (1999) (in Russian).

[30] R.H. Bari, D. Adler, R.V. Lange, Phys. Rev. B 2, 2898 (1970).

[31] L. Didukh, O. Kramar, Yu. Skorenkyy, Yu. Dovhopyaty, Condens. Matter Phys. 8, 825 (2005).

[32] J. Wahle, N. Blümer, J. Schlipf, K. Held, D. Vollhardt, Phys. Rev. B 58, 12749 (1998).

[33] J. Spałek, P. Gopalan, Phys. Rev. Lett. 64, 2823 (1990).

[34] J. Spałek, Phys. Status Solidi B 243, 78 (2006).

[35] A. McCollam, R. Daou, S.R. Julian, J. Flouquet, D. Aoki, Physica B 359-361, 1 (2005). 